\title{
THE RELATIONSHIP BETWEEN ARTHRITIS AND MUSCULAR STRENGTH IN OLDER WOMEN WITH SYMPTOMS OF SARCOPENIA
}

\author{
E.N. Renna ${ }^{1}$, S.G. Slezak ${ }^{1}$, K.B. Mahoney ${ }^{1}$, I.E.Lofgren ${ }^{2}$, D.L. Hatfield ${ }^{1}$, M.J. Delmonico ${ }^{1}$, F. Xu ${ }^{1}$
}

\begin{abstract}
Background: Sarcopenia classification is important for prevention or intervention of sarcopenia in the elderly. However, measures used for the current sarcopenia criteria, including muscular strength, could be impacted by forms of arthritis. Thus, it is crucial to understand the impact arthritis has on sarcopenia status. Objectives: The aim was to investigate if arthritis relates to sarcopenia classification via grip strength or single chair stand in older women. A secondary aim was to assess the relationship between grip strength and upper and lower body strength in those with arthritis. Design: A cross-sectional analysis. Setting and participants: Sixty-one community-dwelling older women (71.9 \pm 4.6 years) from Rhode Island. Measurements: Sarcopenia status was classified using established working definitions. Grip strength was measured using a hand grip dynamometer, chair stands were measured via a single chair stand test, and gait speed was assessed using a four-meter walk test. A segmental multifrequency bioelectrical impedance analysis assessed body composition and arthritis status was based on self-report. Upper and lower body muscular strength were measured using a chest press and leg press one repetition maximum. Results: No associations were observed between arthritis and sarcopenia status $(\mathrm{p}=0.36)$ nor arthritis and upper or lower body muscular strength and grip strength. Conclusions: The results of this study may indicate that arthritis is not associated with sarcopenia status but may affect other measures of muscular strength.
\end{abstract}

Keywords: Sarcopenia, Arthritis, Muscular Strength, Older Women.

\section{Introduction}

Sarcopenia is defined as the loss of muscular strength, functionality, and lean mass with aging (1, 2 ) that is related to functional limitations (3). This is especially a concern among older women since women have a greater life expectancy and are at higher risk for functional disability due to more rapid declines in muscular strength when compared to men (4). As a result of that, sarcopenia also has a great impact on health care with estimated costs for women around \$25.5 billion in the U.S. and continues to increase as the older population increases $(4,5)$. Therefore, it is imperative to screen women for sarcopenia so proper intervention and prevention can be implemented. While a universal sarcopenia classification system is lacking, several national and international organizations have established working classification systems for sarcopenia that include measures of muscular strength, gait speed and lean mass $(1,6-8)$.

1. Department of Kinesiology, University of Rhode Island; 2. Department of Nutrition and Food Sciences, University of Rhode Island

Corresponding Author: Furong Xu, Department of Kinesiology, University of Rhode Island, Independence Square II, 25 West Independence Way, Kingston, RI 02881. Email: fxu2007@uri.edu. Fax: 401-874-4215, Telephone: 401-874-2412
While organizations have various criteria for sarcopenia, muscular strength is often assessed using a grip strength test because it is considered a valid measure to predict overall muscular strength. It is also easier to administer to large populations due to its compact size and portability (1,9-12). Some working definitions use alternative measures to assess muscular strength such as chair stands. The International Working Group uses this measure in their working definition because the ability to rise from a chair is considered an activity of daily living that requires adequate muscular strength $(7,13)$. However, functional limitations such as arthritis could negatively affect muscular strength measurements based on the current sarcopenia working definitions.

Arthritis is a chronic disease characterized by joint stiffness, inflammation, joint deformity, and pain (14). In the U.S., arthritis is the most common cause of disability (15). Between 2013 and 2015, approximately 54.4 million adults were diagnosed with arthritis (15). The symptoms of arthritis (i.e. joint pain, inflammation) could affect one's ability to perform muscular strength tests. Since muscular strength is a key component of all sarcopenia working definitions, it is important to address the potential misclassification of sarcopenia due to the limitations in physical function arthritis may cause (14). Therefore, the purpose of this study was to determine 
Table 1

Inclusion and exclusion criteria used for study recruitment

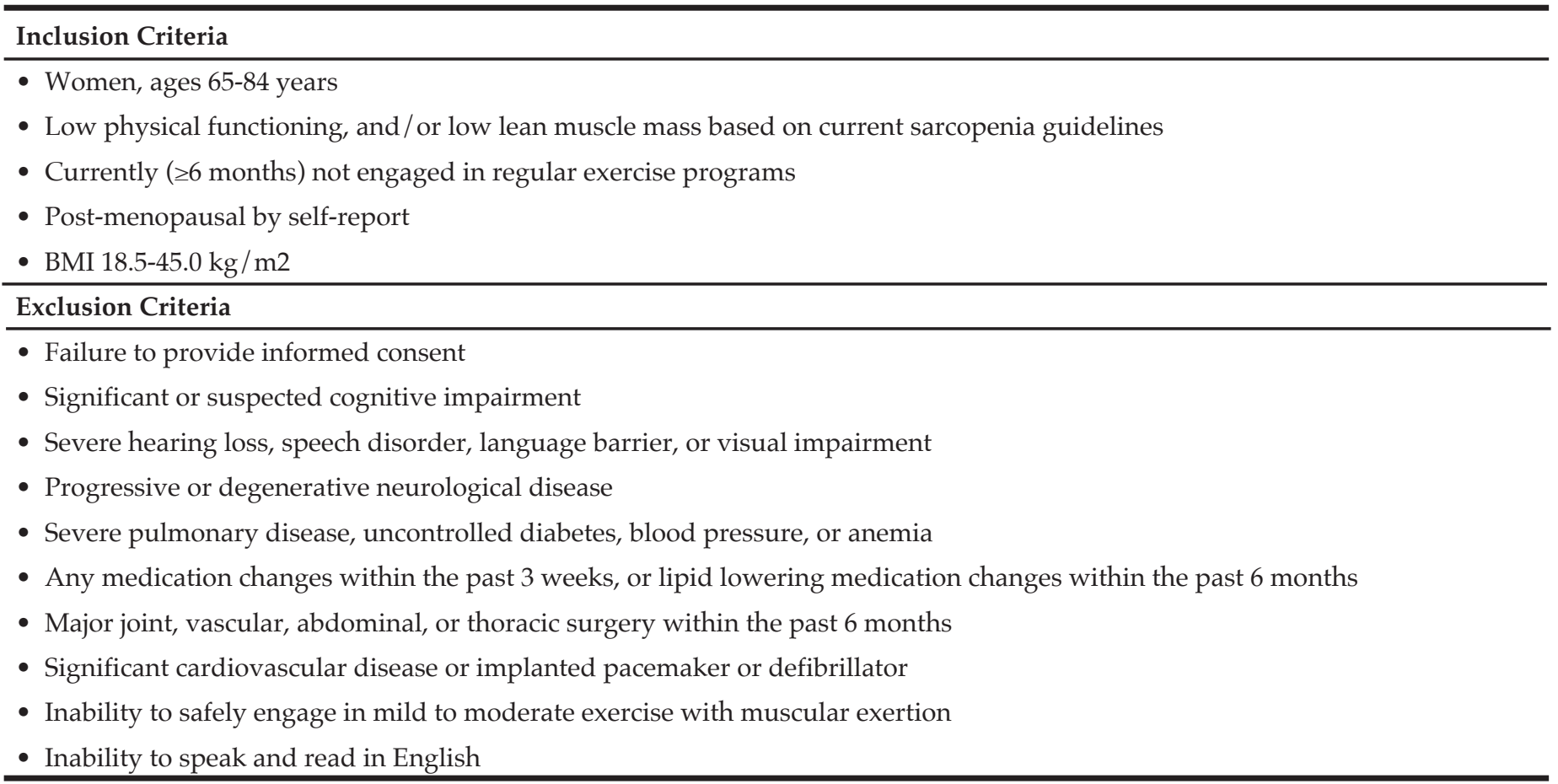

if arthritis is related to sarcopenia classification via grip strength or failure to complete a chair stand in older women. A secondary aim was to assess if grip strength was related to upper and lower body muscular strength in a group of older women with symptoms of sarcopenia by arthritis status.

\section{Materials and methods}

\section{Study Design}

The study utilized a cross-sectional analysis to assess the potential effects of arthritis on sarcopenia status in elderly women who were recruited for Resistance Exercise Study to Reclaim Lean Muscle and Strength (URI RESTORE ME). It was approved by the University of Rhode Island's Institutional Review Board.

\section{Participants}

Participants were recruited from Rhode Island via flyers, community talks and word of mouth, and Figure 1 depicts the flow of participant recruitment. One hundred sixty women were initially phone screened for study eligibility based on the inclusion and exclusion criteria for the study (Table 1). Participants who initially qualified provided written informed consent and were tested for sarcopenia using the European Working Group on Sarcopenia in Older People (EWGSOP) (1), the International Working Group (IWG) (7), and the Foundation for the National Institutes of Health Sarcopenia Project (FNIHSP) criteria $(6,8)$.

\section{Outcome Measures}

\section{Anthropometrics}

Participants had their height, weight, and waist and hip circumferences measured twice. Participants wore surgical scrubs for the waist and hip circumference measurements assessed using standard tape measure with a tensiometer (Gulick Tape Measure, Japan). Weight was measured using a Seca balance beam scale to the nearest $0.1 \mathrm{~kg}$ and height was measured using a Seca wall mounted stadiometer (Seca, Chino, CA) to the nearest 0.1 $\mathrm{cm}$. Both measurements were done without shoes and were performed two times. The average of the two scores was used to calculate each participant's BMI and waist-tohip ratio.

Body composition was also assessed using an InBody 570 SMF-BIA (Biospace Co, Ltd, Korea) device according to the manufacturer's instructions. The SMF-BIA is an accepted valid device used to estimate lean mass and is safe to use in older populations (16). This device has been found to be agreeable to dual energy x-ray absorptiometry (DXA) measurements of appendicular lean mass in this population (17). Measurements were taken at the right and left arms, the right and left legs, 
as well as for the trunk using 8 electrodes specifically placed and 6 different frequencies $(1 \mathrm{kHz}, 5 \mathrm{kHz}, 50$ $\mathrm{kHz}, 250 \mathrm{kHz}, 500 \mathrm{kHz}, 1000 \mathrm{kHz}$ ) which gave a total of 30 impedance measurements for each participant. To standardize the assessment, all participants were asked to be hydrated and fasted for at least four hours and their bladder voided prior to testing.

\section{Sarcopenia Status}

The EWGSOP (1), IWG (7), and FNIHSP $(6,8)$ working definitions were used to determine participants' sarcopenia status based on their performance in a hand grip strength test, a single chair stand, gait speed, and their appendicular lean mass (ALM) as measured using a segmental multi-frequency bioelectrical impedance analysis (SMF-BIA, InBody 570 SMF-BIA, Biospace Co, Ltd, Korea) device. Based on those three working definitions, our criteria for study inclusion were: $<20 \mathrm{~kg}$ for grip strength or inability to complete a single chair stand, a gait speed $<0.8 \mathrm{~m} / \mathrm{s}$, and an ALM $<5.67 \mathrm{~kg} / \mathrm{m} 2$ or $\mathrm{ALM} /$ body mass index $(\mathrm{BMI})<0.512$. Women were then classified as having low muscular strength, low lean mass, low physical functioning (gait speed), all three aspects, or none if no criteria were met. Participants were also considered either grip dependent or nongrip dependent depending on their grip strength score. Participants were considered grip dependent if they only met the muscular strength criteria $(<20 \mathrm{~kg}$ or inability to do a single chair stand) and were considered non-grip dependent if they exceeded the muscular strength criteria or met the other criteria or met no criteria.

\section{Determining Arthritis Presence}

Arthritis was based on self-report noted in participants' phone screening interviews and medical history questionnaires administered during testing sessions. The medical history questionnaire used a "yes/no" question to identify if the participant had arthritis.

\section{Physical Function}

A four-meter gait speed test was used to measure lower extremity functionality (1). Participants walked at a normal walking pace over the four meters for two trials. Participants were hand timed for both trials, and the fastest time was used. Lower extremity function was also assessed using a single chair stand $(7,13)$. Participants were asked to complete a single chair stand unassisted with arms crossed over their chest for one trial. Participants who were unable to stand successfully unassisted were considered to have low muscular strength.

\section{Muscle Function and Strength}

Hand grip strength is used to measure muscular strength (18). It is a key factor in the current working definitions for sarcopenia $(1,8)$, and is a reliable and valid measure for the older adult population (18). Grip strength was assessed using a hand grip dynamometer (Jamar Hydraulic Dynamometer, J.A. Preston Corp., Jackson, MS). Participants were seated with the elbow flexed at 90 degrees. The hand grip dynamometer was adjusted for each participant by ensuring all four fingers had the second knuckle placed flat on the handle. Two trials were completed on each hand with the highest score being recorded in kilograms. Grip strength was measured during testing sessions and at the baseline assessment.

A chest press machine (Cybex International, Inc., Medway, MA) was used to assess maximum upper body strength via a one repetition maximum test (1RM) for each participant employing methods previously published (19). From a seated position, participants had their head, shoulders, and back against a seat back and held onto handles positioned at chest height. Participants then extended their elbows fully and then returned to the starting position to assess their chest press $1 \mathrm{RM}$ (CP1RM). A 1RM for lower body muscular strength (LP1RM) for each participant was determined using a seated leg press machine (Cybex International, Inc., Medway, MA) using methods previously published (20). Briefly, participants were seated and then extended their knees from the starting position ( 90 degrees) by pushing against a platform with their feet until their knees were close to full extension but not locked. For both CP1RM and LP1RM, after a standard warm up, participants completed 3-5 sets of one repetition with a gradual increase in weight and a three-minute rest period between sets until their 1RM was determined.

\section{Other measures}

The Yale Physical Activity Survey (YPAS) questionnaire was administered to evaluate participants' baseline physical activity levels and has been shown to be a valid assessment for determining physical activity levels among older adults (21). This questionnaire was used for describing participants' baseline physical activity levels (21).

The Dietary Screening Tool (DST) was administered to participants at baseline testing to assess their dietary patterns and to determine their level of nutritional risk. There are three levels of nutritional risk that are used to identify if older adults are at risk including: at risk $(<60)$, at possible risk (60-75), and not at risk (>75) (22). This questionnaire was used to describe the baseline characteristics for the participants who partook in baseline testing for the randomized controlled trial. 


\section{Statistical Analysis}

Estimated sample size for this study was determined based on anticipated between-group changes in lean mass rather than change in sarcopenia status by arthritis prevalence. This analysis was part of a larger pilot study to determine the potential for periodized resistance training to impact sarcopenia. The demographic and clinical characteristics for participants are expressed as mean \pm standard deviation for continuous variables and frequencies for categorical variables. A ShapiroWilk test was completed to test for normal distribution. Independent samples t-tests were used to compare those with arthritis to those without arthritis. A Fisher's exact test was used to assess arthritis status and its association to sarcopenia status via multiple sarcopenia definitions for women who were screened $(n=61)$. A Pearson correlation was used to assess the correlation between grip strength, CP1RM, and LP1RM in those with arthritis and those without arthritis from participants who partook in the baseline assessments $(n=25)$. An alpha of $p \leq 0.05$ was used for all statistical analyses and all analyses were performed using SPSS software (IBM SPSS, Version 22, Armonk, NY, 2013).

\section{Table 2}

Characteristics of those with arthritis and without arthritis in participants who completed the testing measurements for the randomized controlled trial

$$
(\mathrm{n}=61)
$$

\begin{tabular}{|c|c|c|c|c|}
\hline & Total & $\begin{array}{c}\text { Arthritis } \\
(\mathrm{n}=35,57.4 \%)\end{array}$ & $\begin{array}{l}\text { No Arthritis } \\
(\mathrm{n}=26,42.6 \%)\end{array}$ & p value \\
\hline Age (years) & $71.9 \pm 4.6$ & $72.0 \pm 4.7$ & $71.7 \pm 4.6$ & 0.780 \\
\hline Weight (kg) & $70.9 \pm 15.2$ & $70.9 \pm 15.1$ & $70.9 \pm 15.6$ & 0.999 \\
\hline Height $(\mathrm{cm})$ & $160.8 \pm 5.6$ & $160.1 \pm 5.3$ & $161.8 \pm 6.0$ & 0.247 \\
\hline $\mathrm{BMI}(\mathrm{kg} / \mathrm{m} 2)^{*}$ & $27.3 \pm 6.0$ & $27.7 \pm 5.8$ & $27.1 \pm 6.0$ & 0.717 \\
\hline$\%$ Body Fat & $40.1 \pm 7.6$ & $40.6 \pm 8.0$ & $39.3 \pm 7.2$ & 0.505 \\
\hline Grip Strength (kg) & $17.6 \pm 4.7$ & $16.6 \pm 4.1$ & $18.9 \pm 5.3$ & 0.063 \\
\hline $\begin{array}{l}\text { Single Chair Stand } \mathrm{n} \\
(\%) \neq, \S\end{array}$ & $60(98.4)$ & $60(98.4)$ & $61(100)$ & $1.00 \neq$ \\
\hline $\begin{array}{l}\text { Gait Speed (meters / } \\
\text { second) }\end{array}$ & $1.02 \pm 0.17$ & $0.99 \pm 0.17$ & $1.06 \pm 0.17$ & 0.131 \\
\hline
\end{tabular}

\section{Results}

Of the 160 women initially interested, 61 women (mean age $71.9 \pm 4.60$ years) qualified for further testing after preliminary phone screening. Of those 61 women, 35 women had arthritis and 26 of those women did not have arthritis. Additionally, 25 women (mean age $72.2 \pm 4.6$ years) out of the 61 women, who qualified for the URI RESTORE ME study, exhibited at least one symptom or sign of sarcopenia defined by various working group definitions and completed all baseline measurements for the URI RESTORE ME study. Of the 25 women, 15 women had arthritis and 10 women did not have arthritis. Tables 2 and 3 describe the sample characteristics by sarcopenia criteria, age, 1RM measures, physical activity scores, and DST scores. There were no significant differences in characteristics between those with arthritis to those without arthritis.

When evaluating the association between arthritis and sarcopenia status via grip strength or a single chair stand, arthritis was not significantly related to sarcopenia status via multiple definitions $(n=61, p=0.36)$. Additionally, when assessing maximal muscular strength measures for the women $(n=25)$ who partook in the baseline testing for the URI RESTORE ME study with arthritis, there were no significant correlations between grip strength and CP1RM ( $r=-0.226, p=0.438)$, grip strength and LP1RM $(\mathrm{r}=-0.118, \mathrm{p}=0.688)$, and CP1RM and LP1RM $(\mathrm{r}=0.389$, $\mathrm{p}=0.152)$. However, for those without arthritis, there was a significant correlation between grip strength and CP1RM ( $r=0.683, p=0.029), C P 1 R M$ and LP1RM ( $r=0.881$, $\mathrm{p}=0.001$ ), but not between grip strength and LP1RM $(\mathrm{r}=0.554, \mathrm{p}=0.097)$.

Figure 1

Study Flow Chart

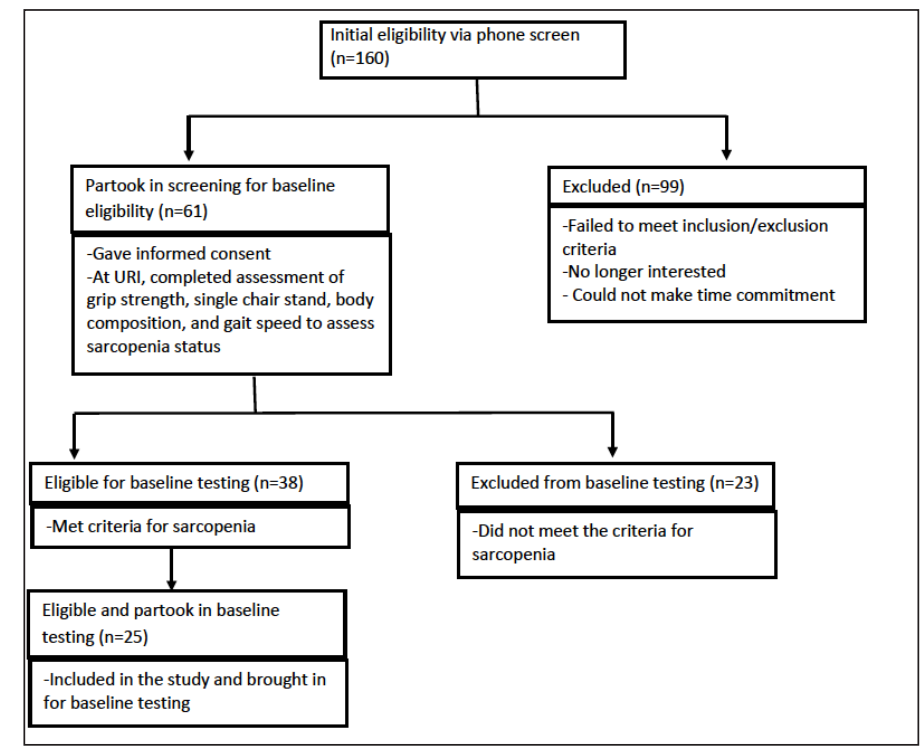

\section{Discussion}

The results of the present study indicate that arthritis was not associated with sarcopenia status in this population of older women based on multiple sarcopenia working definitions. Additionally, our results showed that there was no correlation between grip strength and both upper and lower body muscular strength for those with arthritis.

Contradictory to our findings, a study by Kemmler 
Table 3

Characteristics of those with arthritis and without arthritis for participants who completed baseline measurements for the randomized controlled trial $(n=25)$

\begin{tabular}{|c|c|c|c|c|}
\hline & Total & Arthritis $(n=15,60.0 \%)$ & No Arthritis $(n=10,40.0 \%)$ & P-value \\
\hline Age (years) & $72.2 \pm 4.6$ & $71.9 \pm 4.6$ & $72.9 \pm 4.9$ & 0.619 \\
\hline Weight (kg) & $65.4 \pm 13.5$ & $62.9 \pm 10.9$ & $69.1 \pm 16.6$ & 0.271 \\
\hline Height (cm) & $159.8 \pm 5.2$ & $159.9 \pm 4.8$ & $159.6 \pm 5.9$ & 0.915 \\
\hline $\mathrm{BMI}(\mathrm{kg} / \mathrm{m} 2)^{*}$ & $25.7 \pm 5.9$ & $24.6 \pm 4.3$ & $27.3 \pm 7.6$ & 0.274 \\
\hline Waist to Hip Ratio & $0.87 \pm 0.10$ & $0.88 \pm 0.11$ & $0.86 \pm 0.09$ & 0.745 \\
\hline$\%$ Body Fat† & $37.1 \pm 7.7$ & $36.0 \pm 7.7$ & $38.8 \pm 7.6$ & 0.383 \\
\hline Grip Strength (kg) & $16.6 \pm 3.7$ & $16.6 \pm 4.0$ & $16.7 \pm 3.5$ & 0.949 \\
\hline Single Chair Stand n (\%) | I, \{ & $25(100)$ & $25(100)$ & $25(100)$ & 1.00\{ \\
\hline Gait Speed (meters / second) & $1.05 \pm 0.14$ & $1.07 \pm 0.15$ & $1.02 \pm 0.13$ & 0.359 \\
\hline Chest Press 1 RM (kg) & $17.0 \pm 6.6$ & $15.4 \pm 3.9$ & $19.5 \pm 9.1$ & 0.136 \\
\hline Leg Press 1 RM (kg) & $46.3 \pm 16.1$ & $41.9 \pm 7.5$ & $53.0 \pm 22.9$ & 0.093 \\
\hline Physical Activity (kcals/week)‡ & $7302 \pm 3030$ & $7107 \pm 2663$ & $7574 \pm 3615$ & 0.719 \\
\hline Physical Activity Index & $49.5 \pm 21.9$ & $50.8 \pm 20.1$ & $47.6 \pm 25.2$ & 0.734 \\
\hline $\mathrm{DST}^{*}, \S$ & $69.2 \pm 7.8$ & $70.9 \pm 9.0$ & $67.1 \pm 5.6$ & 0.262 \\
\hline
\end{tabular}

et al (2016) used a cross-sectional analysis to compare osteoarthritis in the hip and lower limb to sarcopenia status in older women and found that those with osteoarthritis were more likely to be sarcopenic than their non-arthritic counterparts (23). However, that study focused only on osteoarthritis in the lower half of the body while we assessed general arthritis to all components of the working definitions (23). Additionally, unlike our present study, that study did not take into consideration other working definitions of sarcopenia and only used the EWGSOP definition. Our study on the other hand, used the EWGSOP, IWG, and FNIHSP working definitions and utilized the single chair stand component of the IWG definition as well as grip strength for measures of muscular strength. The IWG uses this measure because it is an important activity of daily living and requires adequate muscular strength. In our study, we only had one participant who could not perform a chair stand but displayed no other symptoms of sarcopenia despite reporting arthritis. Although we do not know of the kind of arthritis or what joints were affected, it is possible that arthritis could have impaired her ability to perform the chair stand. Poor muscular strength in the lower limbs is known to promote cartilage damage which could progress any current osteoarthritis (24). Future studies need to address both arthritis and sarcopenia together and the potential affects each has on the other. The lack of studies in this area could be due to having high internal validity and therefore not accurately capturing the older adult population.

This study also looked at the relationship between grip strength and upper and lower body muscular strength for those with and without arthritis. This relationship is important because grip strength is often used to assess overall muscular strength as it is more convenient in a clinical setting and easier to administer compared to other muscular strength tests such as 1 RM testing (11). However, the present study found correlations between grip strength and upper and lower body muscular strength for non-arthritis participants but not those with arthritis. This is not consistent with other findings in the literature. Other studies have reported that grip strength and lower body strength as well as grip strength and upper body strength are related, and that using grip strength as a measure for overall muscular strength is an adequate alternative $(11,25,26)$. Yet, those studies only used healthy volunteers while our study evaluated both participants with and without arthritis. Therefore, given the results of our study, it is possible that arthritis played a role in the lack of relationship, and arthritis may have attenuated participants' ability to perform the strength tests (11). This could be due to characteristics of arthritis such as pain, inflammation, and functional limitations $(27,28)$.

This study has limitations and strengths. First, the sample size of our study was small and therefore, the results may not adequately reflect this association. Additionally, we did not focus on one specific type of 
arthritis but rather included all forms of arthritis. Despite these study limitations, this study also has strengths worth noting. First, to our knowledge this is the first study to assess general arthritis and its relationship to sarcopenia status via grip strength and single chair stands in older women with or without symptoms of sarcopenia based on multiple sarcopenia guidelines. While other studies have evaluated specific types of arthritis or used only one working definition in their research. Secondly, this study included a homogenous sample cohort of community-dwelling older women. Finally, this study used measures of muscular strength that have been standardized and validated for older populations $(19,20)$.

\section{Conclusions}

This is the first study to evaluate the impact arthritis has on grip strength or failure to complete a single chair stand in a population of older women for the classification of sarcopenia using multiple sarcopenia guidelines. The present study found that arthritis was not significantly associated with sarcopenia status via grip strength or single chair stand and that there is no significant correlation between grip strength and both upper and lower body muscular strength in older women with arthritis. Although this pilot study adds to the literature, additional studies with a larger sample size and clearly defined arthritis status (i.e. joints affected and type) are needed to determine if these variables are linked and to further explore the lack of the relationship between muscular strength measures.

Funding: This study was funded by a grant from the College of Health Sciences at the University of Rhode Island.

Acknowledgments: : The authors would like to extend their gratitude to all study participants and University student volunteers for their support.

Conflict of interest: The authors declare that there no conflict of interest.

Ethical standard: All procedures performed in this study were in accordance with the ethical standards of the institutional and/ or national research committee and with the 1964 Helsinki declaration and its later amendments or comparable ethical standards.

\section{References}

1. Cruz-Jentoft A, Baeyens J, Bauer J, Boirie Y, Cederholm T, Landi F, et al Sarcopenia: European consensus on definition and diagnosis: Report of the European Working Group on Sarcopenia in Older People. Age Ageing 2010; 39: 412 .

2. Manini TM, Clark BC. Dynapenia and aging: an update. J Gerontol A Biol Sci Med Sci 2012; 67: 28.

3. Beaudart C, Rizzoli R, Bruyère $O$, Reginster J, Biver E. Sarcopenia: burden and challenges for public health. Arch Public Health 2014; 72.

4. Borst SE. Interventions for sarcopenia and muscle weakness in older people. Age Ageing 2004; 33: 548

5. Janssen I, Shepard DS, Katzmarzyk PT, Roubenoff R. The Healthcare Costs of Sarcopenia in the United States. J Am Geriatr Soc 2004; 52: 80.

6. Studenski SA, Peters KW, Alley DE, Cawthon PM, McLean RR, Harris TB, et al. The FNIH Sarcopenia Project: Rationale, Study Description, Conference Recommendations, and Final Estimates. J Gerontol A Biol Sci Med Sci 2014; 69: 547.

7. Fielding RA, Vellas B, Evans WJ, Bhasin S, Morley JE, Newman AB, et al. Sarcopenia: an undiagnosed condition in older adults. Current consensus definition: prevalence, etiology, and consequences. International working group on sarcopenia. J Am Med Dir Assoc 2011; 12: 249.

8. McLean RR, Shardell MD, Alley DE, Cawthon PM, Fragala MS, Harris TB, et al. Criteria for clinically relevant weakness and low lean mass and their longitudinal association with incident mobility impairment and mortality: the foundation for the National Institutes of Health (FNIH) sarcopenia project. J Gerontol A Biol Sci Med Sci 2014; 69: 576.

9. Bohannon RW. Hand-grip dynamometry predicts future outcomes in aging adults. J Geriatr Phys Ther 2008; 31: 3.

10. Cooper C, Fielding R, Visser M, vanLoon L, Rolland Y, Orwoll E, et al. Tools in the assessment of sarcopenia. Calcif Tissue Int 2013; 93: 201.

11. Stark T, Walker B, Phillips JK, Fejer R, Beck R. Hand-held dynamometry correlation with the gold standard isokinetic dynamometry: a systematic review. PM R 2011; 3: 472.

12. Syddall H, Cooper C, Martin F, Briggs R, Aihie Sayer A. Is grip strength a useful single marker of frailty? Age Ageing 2003; 32: 650.

13. Rolland Y, Czerwinski S, Abellan Van Kan G, Morley JE, Cesari M, Onder G, et al. Sarcopenia: its assessment, etiology, pathogenesis, consequences and future perspectives. J Nutr Health Aging 2008; 12: 433.

14. Hootman JM, Helmick CG, Brady TJ. A Public Health Approach to Addressing Arthritis in Older Adults: The Most Common Cause of Disability. Am J Public Health 2012; 102: 426.

15. Barbour KE, Helmick CG, Boring M, Brady TJ. Vital Signs: Prevalence of Doctor-Diangosed Arthritis and Arthritis-Attributable Activity Limitation United States, 2013-2015. 2017; 66: 246-53.

16. Kim M, Kim H. Accuracy of segmental multi-frequency bioelectrical impedance analysis for assessing whole-body and appendicular fat mass and lean soft tissue mass in frail women aged 75 years and older. Eur J Clin Nutr 2013; 67: 395.

17. Mahoney K. Validation of Bioelectrical Impedance Analysis for the Measurement of Appendicular Lean Mass in Older Women. 2016.

18. Roberts HC, Denison HJ, Martin HJ, Patel HP, Syddall H, Cooper C, et al. A review of the measurement of grip strength in clinical and epidemiological studies: towards a standardised approach. Age Ageing 2011; 40: 423.

19. LeBrasseur NK, Bhasin S, Miciek R, Storer TW. Tests of muscle strength and physical function: reliability and discrimination of performance in younger and older men and older men with mobility limitations. J Am Geriatr Soc 2008; 56: 2118.

20. Delmonico MJ, Ferrell RE, Meerasahib A, Martel GF, Roth SM, Kostek MC, et al. Blood pressure response to strength training may be influenced by angiotensinogen A-20C and angiotensin II type I receptor A1166C genotypes in older men and women. J Am Geriatr Soc 2005; 53: 204.

21. Dipietro L, Caspersen CJ, Ostfeld AM, Nadel ER. A survey for assessing physical activity among older adults. Med Sci Sports Exerc 1993; 25: 628

22. Bailey RL, Miller PE, Mitchell DC, Hartman TJ, Lawrence FR, Sempos CT, et al. Dietary screening tool identifies nutritional risk in older adults. Am J Clin Nutr 2009; 90: 177.

23. Kemmler W, Teschler M, Goisser S, Bebenek M, Stengel SV, Bollheimer LC, et al. Prevalence of sarcopenia in Germany and the corresponding effect of osteoarthritis in females 70 years and older living in the community: results of the FORMoSA study. Clin Interv Aging 2015; 10: 1565.

24. De Ceuninck F, Fradin A, Pastoureau P. Bearing arms against osteoarthritis and sarcopenia: when cartilage and skeletal muscle find common interest in talking together. Drug Discov Today 2014; 19: 305.

25. Visser M, Deeg DJ, Lips P, Harris TB, Bouter LM. Skeletal muscle mass and muscle strength in relation to lower-extremity performance in older men and women. J Am Geriatr Soc 2000; 48: 381.

26. Desrosiers J, Hebert R, Bravo G, Dutil E. Comparison of the Jamar dynamometer and the Martin vigorimeter for grip strength measurements in a healthy elderly population. Scand J Rehabil Med 1995; 27: 137.

27. Dedeoğlu M, Gafuroğlu Ü, Yilmaz Ö, Bodur H. The Relationship Between Hand Grip and Pinch Strengths and Disease Activity, Articular Damage, Pain, and Disability in Patients with Rheumatoid Arthritis. Turk J Rheumatol 2013; 28: 69.

28. Scott D, Blizzard L, Fell J, Jones G. Prospective study of self-reported pain, radiographic osteoarthritis, sarcopenia progression, and falls risk in community-dwelling older adults. Arthritis Care Res (Hoboken) 2012; 64: 30. 